\title{
Anatomía de la
}

\section{madera de tres especies de Mimosa (Leguminosae-Mimosoideae) distribuidas en México}

\section{Wood anatomy of three species of Mimosa (Leguminosae-Mimosoideae) occurring in Mexico}

\author{
Susana Adriana Montaño-Arias* , Sara Lucía Camargo-Ricaldel y Rosaura Gretherl
}

\author{
I Departamento de Biología, División de Ciencias Bioló- * Autor de correspondencia. arias_susan@hotmail.com \\ gicas y de la Salud. Universidad Autónoma Metropo- \\ litana-Iztapalapa. Ciudad de México, México. rogg@ \\ xanum.uam.mx, slcr@xanum.uam.mx
}

\section{RESUMEN}

La diversidad morfológica del género Mimosa y su problemática taxonómica han propiciado la búsqueda de caracteres en otras fuentes de evidencia como la anatomía de la madera. Se realizó un estudio comparativo de la anatomía de la madera de tres especies de Mimosa distribuidas en México: M. arenosa, M. lacerata y M. polyantha (Sección Batocaulon); la primera de amplia distribución en la vertiente del Pacífico en México, Nicaragua, Colombia, Venezuela y Brasil; mientras que las otras dos especies son endémicas de México. Del tallo de tres individuos por especie se colectó una troza de $80 \mathrm{~cm}$ de largo, a $80 \mathrm{~cm}$ de distancia del suelo. Se hicieron 25 mediciones de 15 caracteres anatómicos por individuo. Los resultados indican que los caracteres distintivos a nivel específico son: el tipo de porosidad, el tipo y la predominancia del parénquima axial y la presencia o ausencia de traqueidas vasicéntricas y de cristales prismáticos.

PAlabras Clave: anatomía comparada, Batocaulon, características anatómicas, Centroamérica, Sudamérica, taxonomía.

\section{ABSTRACT}

The morphological diversity of the genus Mimosa and its complex taxonomy have led to the search of other sources of characters, such as those provided by the wood anatomy. The aim of this work was to carry out a comparative study of the wood anatomy of three $M i$ mosa species: M. arenosa, M. lacerata y M. polyantha (Section Batocaulon); the first one is widely distributed along the Pacific slopes in Mexico, Nicaragua, Colombia, Venezuela and Brazil; while the other two are endemic to Mexico. One stem fragment of $80 \mathrm{~cm}$ was collected at $80 \mathrm{~cm}$ over the soil, from three plants per species. Twenty-five measurements of 15 wood anatomical characters per individual were made. The results suggest that the distinguishing characters among the studied species are: type of porosity, type and prevalence of axial parenchyma, and the presence or absence of vasicentric tracheids and prismatic crystals.

KEYWORDs: compared wood anatomy, Batocaulon, anatomical characteristics, central America, South America, taxonomy.

\section{INTRODUCCIÓN}

El género Mimosa pertenece a la familia Leguminosae, subfamilia Mimosoideae, ubicado en la tribu Mimoseae. Las leguminosas son una de las seis familias de angiospermas más diversas a nivel mundial y mejor representadas en México (Lewis et al., 2005; Rzedowski, 1991; Sosa y
Dávila, 1994; Sousa et al., 2001). La subfamilia Mimosoideae está conformada por 3270 especies distribuidas principalmente en las regiones tropicales del mundo.

De las Mimosoideae presentes en México, el género Mimosa es el más diverso contando con ca. 110 especies, de las cuales $60 \%$ son endémicas del país (Grether et al., 
1996), por lo que México es considerado el segundo centro de diversificación del género, después de Brasil (Barneby, 1991; Grether, 1978; Simon et al., 2011). Asimismo, el género presenta una gran diversidad de formas de vida, representado tanto por herbáceas como por arbustos, árboles y lianas, con inflorescencias en capítulos o en espigas, y legumbres con las valvas enteras o divididas en artejos; además de establecerse en diversos tipos de vegetación y hábitats (Camargo-Ricalde et al., 2001).

Esta diversidad morfológica ha dado como resultado que la determinación de las especies de Mimosa sea muy compleja (Montaño-Arias, 2010), por lo que varias de ellas han sido objeto de estudios no solamente taxonómicos y sistemáticos (Chehaibar, 1988; Grether, 2000), sino también palinológicos (Caccavari, 1985, 1986, 1987; Chehaibar, 1988; Flores-Cruz et al., 2006), ecológicos (Camargo-Ricalde et al., 2010a, b; MorenoCalles y Casas, 2010; Pavón et al., 2011) y, recientemente, ecoanatómicos (Montaño-Arias et al., 2013). Es relevante señalar que, aunque varios autores (i.e. BaretaKuipers, 1981; Chehaibar y Grether, 1990; Marchiori, 1996; Montaño-Arias, 2010) consideran que el estudio de la anatomía de la madera puede proporcionar caracteres de importancia taxonómica para el género, este aspecto ha sido poco explorado todavía.Los estudios sobre anatomía de la madera de Mimosa corresponden, principalmente, a especies distribuidas en Brasil (i.e. Heringer y De Paula, 1979; Marchiori, 1982, 1985, 1993, 1996; Maccari y Marchiori, 1994; Marchiori y Muñiz, 1997) y algunas en Argentina (i.e. Cozzo y Cristiani, 1950; Cozzo, 1951).

En el caso de las especies de Mimosa distribuidas en México, destacan los estudios de Barajas-Morales y LeónGómez (1989) y de Babos y Cumana (1992), quienes detallaron la anatomía de la madera de M. arenosa (Willd.) Poir.; Chehaibar y Grether (1990), describieron la anatomía de la madera de tres especies de la Sección Mimosa serie Xantiae [=serie Lactifluae]: M. lactiflua Delile ex Benth., M. tricephala Slechtdl. \& Cham. y M. sicyocarpa B. L. Rob. Camargo-Ricalde $(1997,2000)$ y De Oliveira et al. (2006) describieron los caracteres anatómicos de la madera de M. tenuiflora (Willd.) Poir. y Montaño-Arias (2010) determinó los caracteres con valor taxonómico de ocho taxa: M. acantholoba (H. \& B. ex Willd.) Poir. var. eurycarpa (B.L. Rob.) Barneby, M. bahamensis Benth., M. benthamii J.F. Macbr. var. benthamii, M. hexandra M. Micheli., M. leucaenoides Benth., M. tejupilcana R. Grether \& A. Martínez-Bernal, M. tenuiflora y M. texana (A. Gray) Small var. filipes (Britton \& Rose) Barneby, todas ellas pertenecientes a la Sección Batocaulon.

A nivel mundial, se ha descrito la madera de 40 especies de Mimosa, de las ca. 530 especies existentes. Sin embargo, solo algunos trabajos se enfocan al estudio de los caracteres anatómicos de la madera con posible valor taxonómico (Marchiori, 1996; Montaño-Arias, 2010). De las especies de Mimosa que crecen en México, solo se ha estudiado la anatomía de la madera de 13 especies ( $c a$. $12 \%)$, aun cuando se estima que ca. 68 especies (62\%) pudieran ser maderables (Barneby, 1991; Grether et al. 1996; Sousa et al. 2001, 2003).

\section{OBJETIVo}

El objetivo de este estudio fue describir y analizar en forma comparativa, la anatomía de la madera de tres especies de Mimosa: M. arenosa, M. lacerata Rose y $M$. polyantha Benth., pertenecientes a la Sección Batocaulon, con la finalidad de determinar aquellos caracteres de valor taxonómico que contribuyan al conocimiento del género.

\section{MATERIALES Y MÉTODOS}

\section{Selección de especies y colecta de material}

Se eligieron tres especies arbóreas de Mimosa, de amplia distribución en México que habitan en zonas secas: $M$. arenosa, M. lacerata y M. polyantha. Se colectaron tres árboles de talla similar, por especie. Para cada sitio de colecta, se registraron las coordenadas geográficas y datos de los individuos (altura y diámetro del tronco a la altura del pecho, DAP). Del tallo de cada uno de los tres individuos, por especie, se colectó una troza de $80 \mathrm{~cm}$ de largo, a $80 \mathrm{~cm}$ de distancia del suelo. Los ejemplares de referencia y las tablillas correspondientes están depositados en el 
Herbario Metropolitano (UAMIZ), de la Universidad Autónoma Metropolitana, Unidad Iztapalapa (Tabla 1).

\section{Trabajo de Laboratorio}

Con un micrótomo de deslizamiento American Optical, modelo 860, se cortaron secciones transversales, tangenciales y radiales, con grosor de $20 \mu \mathrm{m}$; se tiñeron con safranina-verde rápido y se montaron en resina sintética (Johansen, 1940). Además, se colocaron astillas de la sección radial en un tubo de ensayo con una mezcla, a partes iguales, de ácidos acético, láctico y nítrico y glicerina, dejándolos reposar por 1-2 semanas. Una vez disociado el material, este se lavó con agua destilada y se hicieron preparaciones temporales para cuantificar la longitud de los elementos de vaso, de las fibras y de las traqueidas vasicéntricas. Se realizaron 25 mediciones para cada característica, por individuo, por especie.

\section{Descripción de los caracteres anatómicos}

La clasificación de los caracteres cuantitativos se hizo con base en la media (IAWA, 1989), la descripción de los radios según Kribs (1968) y los cristales conforme a Chattaway (1956). El número de vasos por milímetro cuadrado se contabilizó tomando en cuenta a los vasos solitarios y agrupados. Los vasos agrupados se contaron de manera individual, cada uno como solitario. El grosor de la pared del elemento de vaso se midió en corte transversal; mientras que se siguió la clasificación propuesta por MontañoArias (2010) para medir el diámetro completo de la punteadura intervascular y el diámetro del lumen de la punteadura en corte tangencial, el cual generalmente se describe como pequeño, mediano y grande. Para la longitud del elemento del vaso y de fibras se utilizó la clasificación propuesta por IAWA (1989). Asimismo, para la longitud del cordón de parénquima y traqueidas vasicéntricas, se utilizó la clasificación propuesta para longitud de fibras de IAWA (1989). Todas las mediciones se realizaron utilizando un microscopio óptico marca Zeiss, modelo Axiostar-Plus.

\section{Análisis estadísticos}

Se evaluó la existencia de diferencias estadísticamente significativas entre los caracteres analizados intra- e interespecíficamente (Sokal y Rohlf, 1995); los datos obtenidos se examinaron por medio de un análisis de varianza (ANOVA, $P \leq 0.05$ ), seguido por la prueba de Tukey-Kramer

Tabla 1. Ubicación de los sitios de colecta de Mimosa arenosa, M. lacerata y M polyantha (Leguminosae-Mimosoideae).

\begin{tabular}{|c|c|c|c|c|}
\hline Especie & Estado & $\begin{array}{l}\text { Coordenadas } \\
\text { (Altitud m snm) }\end{array}$ & $\begin{array}{c}\text { Tipo de } \\
\text { vegetación }\end{array}$ & $\begin{array}{l}\text { UAMIZ No. Registro } \\
\text { Ejemplares / Tablilla }\end{array}$ \\
\hline M. arenosa & Oaxaca & $\begin{array}{l}16^{\circ} 24.503^{\prime} \mathrm{N} ; \\
94^{\circ} 47.620^{\prime} \mathrm{W} \\
\text { (350 m snm) }\end{array}$ & $\begin{array}{l}\text { Bosque tropical } \\
\text { caducifolio }\end{array}$ & $64627,64628,64629 / 285$ \\
\hline M. lacerata & Puebla & $\begin{array}{c}18^{\circ} 41^{\prime} 31^{\prime \prime} \mathrm{N} ; \\
97^{\circ} 24^{\prime} \mathrm{O} 1.3^{\prime \prime} \mathrm{W} \\
(2,232 \mathrm{~m} \mathrm{snm})\end{array}$ & Matorral xerófilo & 63910, 63911, 63919 / 217 \\
\hline M. polyantha & Puebla & $\begin{array}{c}18^{\circ} 15^{\prime 2} 23.7^{\prime \prime} \mathrm{N} ; \\
97^{\circ} 09^{\prime} 03.3^{\prime \prime} \mathrm{W} \\
(1,140 \mathrm{~m} \mathrm{snm})\end{array}$ & Matorral xerófilo & $71059,71060,71061 / 220$ \\
\hline
\end{tabular}

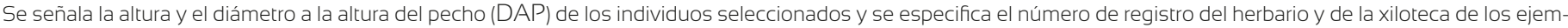
plares de referencia y de las tablillas depositados en el Herbario Metropolitano (UAMIZ). 
$(P \leq 0.05)$. Los análisis estadísticos se llevaron a cabo mediante el paquete estadístico NCSS (Hintze, 2001).

\section{RESULTADOS}

\section{Porosidad}

Mimosa arenosa y $M$. polyantha presentan porosidad difusa; mientras que M. lacerata tiene porosidad anular. En M. arenosa, los anillos de crecimiento están delimitados por una banda de fibras engrosadas y por una banda de parénquima; en M. polyantha, los anillos de crecimiento solo están delimitados por fibras engrosadas; mientras que en $M$. lacerata, los anillos de crecimiento se encuentran delimitados por una banda de fibras y vasos de mayor diámetro, que corresponden a la madera temprana (Fig. 1 a, b, c).

La madera de Mimosa arenosa y M. polyantha presenta vasos numerosos (>10 vasos $\left./ \mathrm{mm}^{2}\right)$. M. arenosa presenta el mayor número de vasos $/ \mathrm{mm}^{2}$, lo que la distingue de M. polyantha; sin embargo, no presenta diferencia significativa con esta última (Tabla 2 y Fig. 1 a y c). En el caso de M. lacerata, la madera temprana presenta pocos $(<10)$ vasos $/ \mathrm{mm}^{2}$; mientras que la madera tardía tiene vasos numerosos (Tabla 3 y Fig. 1 a, b). Las tres especies presentan vasos agrupados; no obstante, en $M$. arenosa y
M. polyantha se observan de dos a tres vasos agrupados, mientras que en la madera temprana y en la tardía de $M$. lacerata, únicamente se observan dos vasos agrupados.

\section{Descripción de los elementos de vaso}

Los elementos de vaso que poseen diámetro tangencial pequeño y pared delgada se observan en Mimosa arenosa y M. polyantha, sin mostrar diferencias estadísticamente significativas (Tabla 2, Fig. 1 a, b, c). Los vasos de la madera temprana y tardía de M. lacerata también presentan un diámetro tangencial pequeño y son de pared delgada; cabe mencionar que los elementos de vaso de la madera temprana son $c a .50 \%$ más grandes que los de la madera tardía (Tabla 3 ).

Respecto a la longitud de los elementos de vaso, las tres especies estudiadas presentan elementos de vaso cortos; en este rango, Mimosa arenosa tiene los elementos de vaso de mayor longitud; aunque, los de la madera tardía de M. lacerata son de longitud similar a los elementos de vaso de M. arenosa (Tablas 2 y 3 ).

Las tres especies presentan elementos de vaso con placas de perforación simple (Fig. 1 g, h, i), punteaduras intervasculares areoladas, alternas y ornamentadas. No se observan diferencias significativas en cuanto al diá-

Tabla 2. Elementos de vaso de dos especies de Mimosa: M. arenosa y M. polyantha (Leguminosae-Mimosoideae); ambas presentan porosidad difusa.

\begin{tabular}{ccccccc}
\hline \multirow{2}{*}{ Variables } & Especies & Media \pm desviación & Valor & \multicolumn{2}{c}{ Valor } & \multicolumn{2}{c}{ ANOVA } \\
\cline { 6 - 7 } & & estándar & mínimo & máximo & $F$ & $P$ \\
\hline \multirow{2}{*}{ Vasos $/ \mathrm{mm}^{2}$} & M. arenosa & $36.05 \pm 2.54^{\mathrm{a}}$ & 21 & 54 & & \\
& M. polyantha & $32.15 \pm 2.08^{\mathrm{a}}$ & 21 & 47 & 4.24 & 0.108495 \\
Ø tangencial & M. arenosa & $69.03 \pm 5.0^{\mathrm{a}}$ & 35 & 115 & & \\
del vaso $(\mu \mathrm{m})$ & M. polyantha & $67.40 \pm 6.07^{\mathrm{a}}$ & 50 & 105 & 0.14 & 0.728115 \\
Grosor de la & M. arenosa & $6.60 \pm 0.17^{\mathrm{a}}$ & 5 & 10 & & \\
pared $(\mu \mathrm{m})$ & M. polyantha & $6.33 \pm 0.21^{\mathrm{a}}$ & 5 & 10 & 2.91 & 0.163279 \\
Longitud de los & M. arenosa & $162.63 \pm 13.0^{\mathrm{a}}$ & 100 & 237 & & \\
elementos vaso $(\mu \mathrm{m})$ & M. polyantha & $125.90 \pm 5.0^{\mathrm{b}}$ & 50 & 187.5 & 21.19 & $0.010008^{*}$ \\
\hline
\end{tabular}

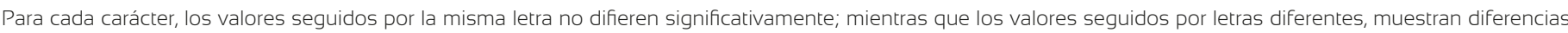
significativas $(P<0.05)$. $\varnothing=$ Diámetro. *Diferencias estadísticamente significativas. 


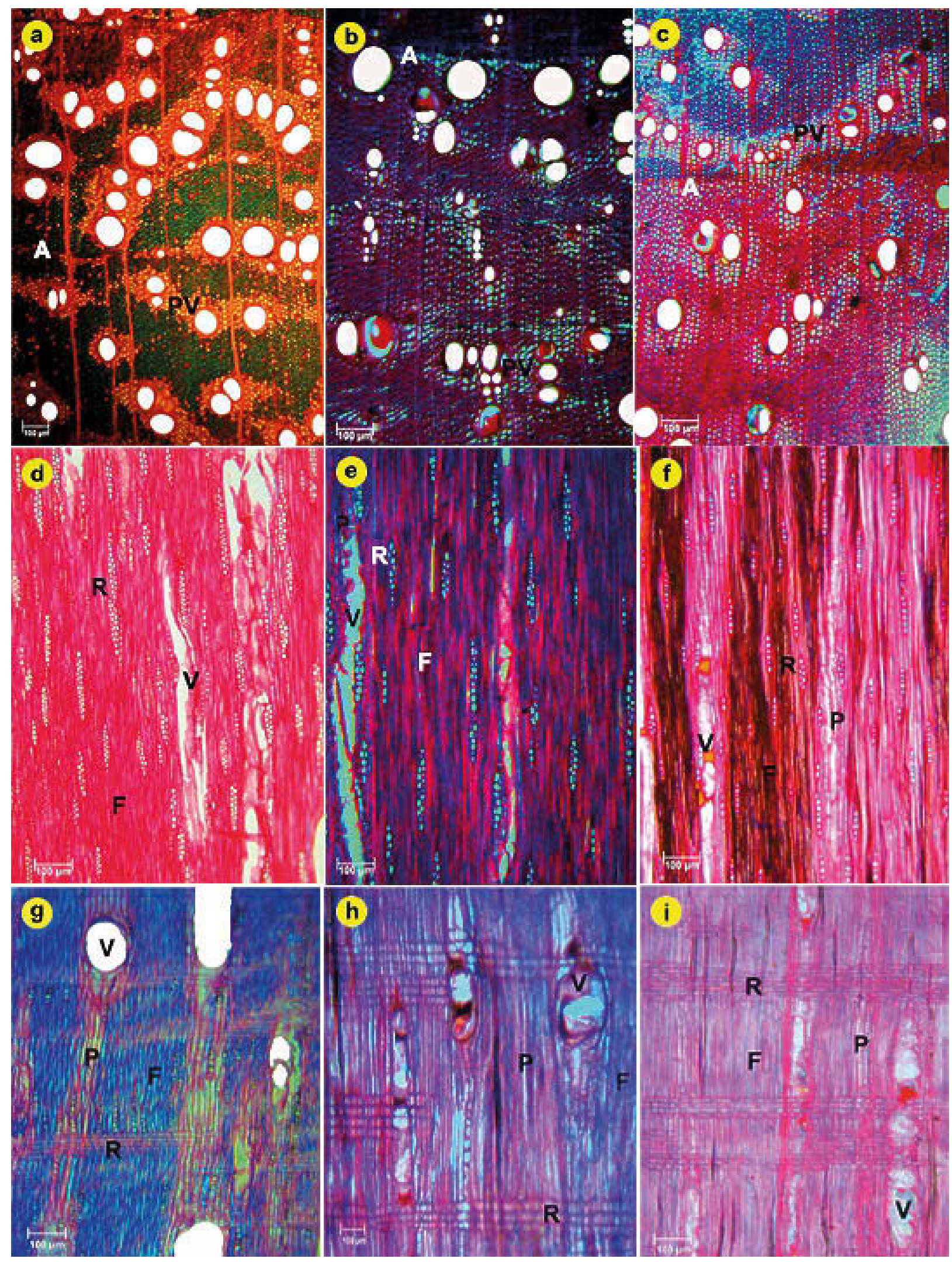

Figura 1. Anatomía de la madera de tres especies del género Mimosa. a, d, g. M. arenosa; b, e, h. M. lacerata; y c, f, i. M. polyantha. A: Anillo de crecimiento, F: Fibras, P: Parénquima, PV: Parénquima vasicéntrico, R: Radios, V: Vaso. 
Tabla 3. Elementos de vaso de Mimosa lacerata (Leguminosae-Mimosoideae); presenta porosidad anular.

\begin{tabular}{|c|c|c|c|c|c|c|}
\hline \multirow{3}{*}{ Variables } & \multicolumn{2}{|c|}{ Media \pm desviación estándar } & \multicolumn{2}{|c|}{ Valor mínimo } & \multicolumn{2}{|c|}{ Valor máximo } \\
\hline & Madera & Madera & Madera & Madera & Madera & Madera \\
\hline & Temprana & Tardía & Temprana & Tardía & Temprana & Tardía \\
\hline Vasos/mm² & $9.98 \pm 2.0$ & $16.70 \pm 6.0$ & 4 & 6 & 23 & 29 \\
\hline $\begin{array}{l}\varnothing \text { tangencial } \\
\text { del vaso }(\mu \mathrm{m})\end{array}$ & $82.63 \pm 4.55$ & $42.33 \pm 5.68$ & 45 & 12.5 & 125 & 67.5 \\
\hline $\begin{array}{c}\text { Grosor de } \\
\text { la pared }(\mu \mathrm{m})\end{array}$ & $7.20 \pm 0.82$ & $5.71 \pm 0.36$ & 5 & 2.5 & 10 & 7.5 \\
\hline $\begin{array}{l}\text { Longitud de los } \\
\text { elementos vaso }(\mu \mathrm{m})\end{array}$ & $120.7 \pm 5.63$ & $171.2 \pm 34.0$ & 50 & 87.5 & 167.5 & 250 \\
\hline
\end{tabular}

$\varnothing=$ Diámetro

metro de la punteadura intervascular y al diámetro del lumen de la punteadura (Tabla 4).

\section{Parénquima axial}

Las tres especies presentan parénquima paratraqueal; en forma adicional, Mimosa lacerata presenta parénquima apotraqueal escaso. En M. arenosa, el parénquima es principalmente aliforme confluente, uniendo de dos a cuatro vasos, seguido del aliforme y, en menor proporción, vasicéntrico. M. lacerata tiene parénquima vasicéntrico principalmente, aunque también presenta parénquima unilateral. En M. polyantha, se observa parénquima, principalmente vasicéntrico, seguido del aliforme y, en menor proporción, aliforme confluente, uniendo de dos a cinco vasos (Fig. 1 a, b, c).

En las tres especies, las células del parénquima axial forman cordones cortos; en este rango, Mimosa lacerata tiene los cordones más largos, aunque, no hay diferencias significativas con los de M. arenosa y M. polyantha (Tabla 4). En el caso de estas dos últimas, los cordones están constituidos por una célula; mientras que en M. lacerata están integrados por dos.

Por otro lado, únicamente las células del parénquima axial de $M$. arenosa presentan de 11 a 14 cristales prismáticos de oxalato de calcio, cada uno de ellos ubicado en una cámara.

\section{Radios}

Las tres especies presentan radios numerosos (cinco radios/mm), biseriados; sin embargo, en Mimosa arenosa y $M$. lacerata también se observan radios triseriados. Los radios son homogéneos, están compuestos por células procumbentes, son bajos y finos. Estadísticamente, $M$. arenosa y M. lacerata tienen los radios más altos y gruesos; mientras que en M. polyantha son más bajos y delgados (Tabla 4 y Fig. 1 d-i).

\section{Fibras}

En las tres especies se observan fibras libriformes, de longitud corta, diámetro fino y pared delgada. Las fibras de Mimosa arenosa tienen de cinco a ocho cristales prismáticos, cada uno en una cámara. No se observan diferencias significativas entre especies, ni a nivel intra-específico (Tabla 4 y Fig. 1 d-i).

Solamente Mimosa polyantha presenta traqueidas vasicéntricas de longitud corta $(669 \mu \mathrm{m})$, diámetro fino $(7.4 \mu \mathrm{m})$ y pared delgada $(5 \mu \mathrm{m})$.

\section{DISCUSIÓN}

Relevancia taxonómica. La madera de las tres especies de Mimosa estudiadas, presenta características anatómicas (i.e. porosidad, placas de perforación simple, punteaduras 
TAвla 4. Resultados de la estadística descriptiva y de los análisis de varianza (ANOVA), correspondientes a radios y fibras de las tres especies de Mimosa (Leguminosae-Mimosoideae) estudiadas.

\begin{tabular}{|c|c|c|c|c|c|c|}
\hline \multirow{2}{*}{ Variables } & \multirow{2}{*}{ Especies } & \multirow{2}{*}{$\begin{array}{c}\text { Media } \pm \text { desviación } \\
\text { estándar }\end{array}$} & \multirow{2}{*}{$\begin{array}{l}\text { Valor } \\
\text { mínimo }\end{array}$} & \multirow{2}{*}{$\begin{array}{c}\text { Valor } \\
\text { máximo }\end{array}$} & \multicolumn{2}{|c|}{ ANOVA } \\
\hline & & & & & $F$ & $P$ \\
\hline \multirow{3}{*}{$\begin{array}{l}\varnothing \text { de la punteadura } \\
\text { intervascular }(\mu \mathrm{m})\end{array}$} & M. arenosa & $5.9 \pm 0.4$ & 5 & 7.5 & & \\
\hline & M. lacerata & $5.9 \pm 0.36$ & 5 & 7.5 & 0.18 & 0.842421 \\
\hline & M. polyantha & $6.1 \pm 0.62$ & 5 & 7.5 & & \\
\hline \multirow{3}{*}{$\begin{array}{c}\varnothing \text { del lumen de la punteadura } \\
\text { intervascular }(\mu \mathrm{m})\end{array}$} & M. arenosa & $3.4 \pm 0.4$ & 2.5 & 5 & & \\
\hline & M. lacerata & $3.43 \pm 0.40$ & 2.5 & 5 & 0.21 & 0.819555 \\
\hline & M. polyantha & $3.63 \pm 0.61$ & 2.5 & 5 & & \\
\hline \multirow{3}{*}{$\begin{array}{l}\text { Longitud del cordón del } \\
\text { parénquima }(\mu \mathrm{m})\end{array}$} & M. arenosa & $170.87 \pm 5.78$ & 100 & 225 & & \\
\hline & M. lacerata & $207.07 \pm 37.73$ & 60 & 385 & 3.32 & 0.106750 \\
\hline & M. polyantha & $149.93 \pm 9.92$ & 100 & 225 & & \\
\hline \multirow{3}{*}{ Radios/mm } & M. arenosa & $5.81 \pm 0.13$ & 3 & 8 & & \\
\hline & M. lacerata & $5.60 \pm 0.36$ & 1 & 3 & 3.06 & 0.121588 \\
\hline & M. polyantha & $4.91 \pm 0.72$ & 1 & 7 & & \\
\hline \multirow{3}{*}{ Altura de radios $(\mu \mathrm{m})$} & M. arenosa & $133.67 \pm 4.11^{\mathrm{a}}$ & 55 & 250 & & \\
\hline & M. lacerata & $145.80 \pm 31.91^{\mathrm{a}}$ & 75 & 290 & 8.25 & $0.018987^{*}$ \\
\hline & M. polyantha & $84.53 \pm 10.66^{b}$ & 35 & 130 & & \\
\hline \multirow{3}{*}{ Anchura de radios ( $\mu \mathrm{m})$} & M. arenosa & $20.87 \pm 1.47$ & 15 & 30 & & \\
\hline & M. lacerata & $24.33 \pm 3.52$ & 15 & 35 & 5.68 & $0.041220^{*}$ \\
\hline & M. polyantha & $17.40 \pm 2.11$ & 10 & 25 & & \\
\hline \multirow{3}{*}{ Longitud de fibras ( $\mu \mathrm{m})$} & M. arenosa & $542.13 \pm 17.46$ & 430 & 890 & & \\
\hline & M. lacerata & $536.13 \pm 78.62$ & 360 & 850 & 0.08 & 0.920421 \\
\hline & M. polyantha & $565.20 \pm 113.49$ & 290 & 860 & & \\
\hline \multirow{3}{*}{$\varnothing$ del lumen de la fibra ( $\mu \mathrm{m})$} & M. arenosa & $6.73 \pm 0.85$ & 5 & 10 & & \\
\hline & M. lacerata & $7.0 \pm 0.62$ & 5 & 10 & 1.94 & 0.224286 \\
\hline & M. polyantha & $7.77 \pm 0.47$ & 5 & 10 & & \\
\hline \multirow{3}{*}{$\begin{array}{l}\text { Grosor de la pared de la fibra } \\
\qquad(\mu \mathrm{m})\end{array}$} & M. arenosa & $5.13 \pm 0.15$ & 5 & 7.5 & & \\
\hline & M. lacerata & $5.30 \pm 0.36$ & 2.5 & 7.5 & 1.33 & 0.333486 \\
\hline & M. polyantha & $5.0 \pm 0$ & 5 & 5.0 & & \\
\hline
\end{tabular}

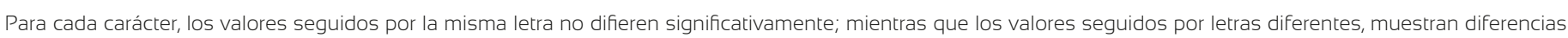
significativas $(P<0.05)$. $\varnothing=$ Diámetro. *Diferencias estadísticamente significativas.

areoladas, alternas y ornamentadas y radios homogéneos, entre otras) similares a las ya descritas para otras especies del género distribuidas tanto en Argentina (Cozzo y Cristiani, 1950; Cozzo, 1951) como en Brasil (Record y Hess,
1943; Tortorelli, 1956; Metcalfe y Chalk, 1979; Heringer y De Paula, 1979; Marchiori, 1982, 1985; Maccari y Marchiori, 1994; Marchiori, 1996; Marchiori y Muñiz, 1997; Fabrowski et al., 2005; De Oliveira et al., 2006; 
Evans et al., 2006) y en México (Barajas-Morales y LeónGómez, 1989; Camargo-Ricalde, 2000; Chehaibar y Grether, 1990; Montaño-Arias, 2010).

La porosidad anular de la madera de Mimosa lacerata permite distinguirla claramente de la madera de $M$. arenosa y $M$. polyantha, las cuales presentan porosidad difusa. Cabe mencionar que la porosidad anular también ha sido observada en otras especies que se distribuyen en climas cálidos subhúmedos como M. acantholoba var. eurycarpa, y en climas secos como $M$. benthamii var. benthamii y M. texana var. filipes (Montaño-Arias, 2010). En el caso de la porosidad difusa, esta se considera un carácter predominante en las Mimosoideae (Evans et al., 2006) y ha sido observada en varias especies de Mimosa como M. detinens Benth., M. hassleriana Chodat, M. uliginosa Chodat \& Hassl., M. uruguensis H. et A. (Cozzo, 1951); M. eriocarpa Benth. (Carnieletto y Marchiori, 1993); M. incana (Spreng.) Benth. (Marchiori, 1996) y M. sparsa Benth. (Maccari y Marchiori, 1994).

Otro carácter utilizado para diferenciar entre especies son las punteaduras alternas y areoladas, las cuales también han sido observadas en otras 19 especies de Mimosa, entre ellas M. bahamensis, M. tejupilcana, M. tenuiflora, M. texana var. filipes (Montaño-Arias, 2010); por lo que se coincide con Baretta-Kuipers (1981) y Marchiori (1996) en el sentido de que las punteaduras intervasculares tienen relevancia taxonómica a nivel genérico y carecen de ella a nivel infragenérico. Además, estas punteaduras son ornamentadas en las tres especies estudiadas; igualmente, este carácter ha sido observado en otras 16 especies de Mimosa como M. cruenta Benth. (Marchiori, 1985), M. eriocarpa (Carnieletto y Marchiori, 1993), M. incana (Marchiori, 1996), M. sparsa (Maccari y Marchiori, 1994), entre otras; cabe mencionar que en el estudio sobre la madera de $M$. detinens, $M$. hassleriana, M. uruguensis, M. pigra L., M. scabrella Benth. (Cozzo, 1951), entre otras, no se menciona si las punteaduras son ornamentadas, por lo que todavía tendrá que evaluarse en un mayor número de especies para conocer su relevancia en la taxonomía del género Mimosa.
Por otro lado, Baretta-Kuipers (1981) determinó que el parénquima axial es un carácter muy variable, aún en la misma especie; aunque, Cozzo (1951) había señalado que la abundancia del parénquima axial es útil para diferenciar entre especies. En este estudio, Mimosa arenosa presentó parénquima aliforme confluente abundante, lo que la distingue de $M$. lacerata y de $M$. polyantha; sin embargo, este carácter ha sido observado en otras especies como M. leucaenoides, M. tejupilcana y M. tenuiflora (Montaño-Arias, 2010). Asimismo, en este estudio, M. lacerata es la única especie que presenta parénquima unilateral, pero este carácter ya fue observado en M. bahamensis (Montaño-Arias, 2010).

Los radios, al igual que el parénquima axial, son caracteres relacionados con aspectos evolutivos de las plantas (Aguilar-Rodríguez y Barajas-Morales, 2005; Kribs, 1935). En este caso, los radios homogéneos han sido observados en 95\% de las especies de Mimosa estudiadas, de manera que es factible considerarlos como característicos del género, coincidiendo con el resto de la subfamilia Mimosoideae (Baretta-Kuipers, 1981; Evans et al., 2006).

Asimismo, Zindler-Frank (1987) y Espinoza de Pernía et al. (1998) señalan que la presencia de cristales prismáticos es común en la familia Leguminosae, así como en la subfamilia Mimosoideae (Evans et al., 2006; MontañoArias, 2010- i.e. Acacia spp. Mimosa spp., y Prosopis spp., entre otros) y en las subfamilias Caesalpinoideae y Papilionoideae (Evans et al., 2006); este carácter fue observado en el parénquima axial de las tres especies estudiadas y también se ha encontrado en el parénquima axial y fibras de M. benthamii var. benthamii, M. hexandra, $M$. leucaenoides, M. tenuiflora y M. texana var. filipes (Montaño-Arias, 2010). Cabe mencionar que de acuerdo con Evans et al. (2006), la existencia de cristales en los radios es un evento raro, que solo ha sido observado en Prosopis farcta (Banks \& Sol.) J.F. Macbr. y Acacia horrida Willd. En Mimosa, la presencia de cristales en los radios ha sido observada en M. benthamii var. benthamii, M. hexandra y $M$. leucaenoides, pero únicamente en $M$. benthamii var. benthamii se han encontrado cristales arenosos en las 
células radiales (Montaño-Arias, 2010) por lo que su presencia, hasta ahora, es distintiva de este último taxon.

Otra característica de la madera, común en la subfamilia Mimosoideae, es la presencia de fibras septadas (Baretta-Kuipers, 1981; Evans et al., 2006) que permiten diferenciarla de las otras dos subfamilias de leguminosas. No obstante, en este caso es importante señalar que las especies estudiadas, no presentaron fibras septadas, de manera que este carácter debe evaluarse conforme se incrementen los estudios de la madera de otras Mimosoideae.

Relevancia ecoanatómica. Mimosa arenosa y $M$. polyantha crecen en sitios con climas secos; la primera es característica de bosques tropicales caducifolios y la última crece, principalmente, en matorrales xerófilos y, en ocasiones, en el bosque tropical caducifolio; sin embargo, ambas especies presentan madera con porosidad difusa, lo que coincide con los resultados de Gilbert (1940, en Moglia y Giménez, 1998), al considerarla una característica distintiva de especies que habitan lugares con escasez de agua.

Aunque la porosidad difusa ha sido observada en otras especies arbóreas y arbustivas de Mimosa que habitan en sitios de clima cálido como: M. adpressa H. et A., M. bimucronata (DC) OK., M. cruenta, M. detinens, $M$. hassleriana, M. ostenii Speg. ex Burk., M. pigra, M. polycarpa Kunth, M. polycarpa var. spegazzini (Pirotta) Burk., M. scabrella, M. uliginosa y M. uruguensis (Cozzo,1951; Marchiori, 1985), M. schomburgkii Benth. (Record y Hess, 1943; Heringer y De Paula, 1979), M. artemisiana Heringer y De Paula (Heringer y De Paula, 1979), no es posible hacer la asociación con la cantidad de humedad presente en sus hábitats, debido a la carencia de información sobre los sitios de colecta.

Otra característica de importancia ecológica son las punteaduras ornamentadas presentes en las tres especies estudiadas; al respecto, Carlquist (1982) y Choat et al. (2004) consideran que la presencia de punteaduras intervasculares ornamentadas proporciona una ventaja ecológica para las especies, al impedir la entrada de burbujas de aire al elemento de vaso, provocadas por la pérdida de la presión del agua.
Por otra parte, Mimosa polyantha es la única de las especies aquí estudiadas, con traqueidas vasicéntricas; sin embargo, este carácter también ha sido observado en $M$. benthamii var. benthamii, $M$. hexandra y M. texana var. filipes (Montaño-Arias, 2010), y en M. scabrella y M. adpressa (Cozzo y Cristiani, 1950). La relevancia de las traqueidas vasicéntricas radica en que le confiere al xilema secundario una mayor seguridad en la conducción de agua (Montaño-Arias et al., 2013), ya que las traqueidas vasicéntricas funcionan como un sistema de conducción subsidiario o alternativo que cumple la misma función que los vasos agrupados (Moglia y Giménez, 1998), volviendo a los taxa más competitivos.

\section{CONCLUSIONES}

Los caracteres descritos y analizados de la anatomía de la madera de las tres especies de Mimosa estudiadas: $M$. arenosa, M. lacerata y M. polyantha, comparados con las $c a .40$ descripciones correspondientes a otras especies del género, revelan que la anatomía de la madera es bastante uniforme, de manera que la madera del género Mimosa se define por presentar porosidad anular o difusa, vasos con diámetro pequeño o mediano, vasos agrupados y múltiples radiales, que son variables en número. Presenta punteaduras alternas y areoladas, la mayoría de los taxa tienen punteaduras ornamentadas y rara vez, son coalescentes (i.e. M. bimucronata). Las punteaduras radio-vasculares son iguales a las punteduras intervasculares. El parénquima axial es principalmente paratraqueal y, en menor proporción, apotraqueal. El parénquima paratraqueal es vasicéntrico, aliforme, aliforme confluente y/o unilateral; en ocasiones, se presenta en forma de bandas concéntricas irregulares (i.e. M. hexandra). Los cordones de parénquima van de 1 a 8 células. La anchura de los radios es variable, pueden ser uniseriados, biseriados y/o triseriados. Los radios son principalmente homogéneos, rara vez heterogéneos. Las fibras son libriformes, sin septos, con paredes delgadas o gruesas. A veces, se pueden presentar traqueidas vasicéntricas. Los cristales de oxalato de calcio son muy comu- 
nes en el parénquima axial y en fibras, y es poco común observarlos en los radios (i.e. M. hexandra, M. leucaenoides); ocasionalmente, en los radios se presenta arena cristalina (i.e. M. benthamii var. benthamii). Las gomas son comunes en los elementos de vaso.

Por otra parte, las características que definen a la madera del género Mimosa son propias de especies resistentes a la sequía; de manera que, se puede sugerir que los taxa presentes en lugares templados (i.e. M. tejupilcana) podrían sobrevivir en ambientes secos, ya que mantienen una estructura adaptada para asegurar la conducción de agua y el uso eficaz de ella.

\section{RECONOCIMIENTOS}

La primera autora agradece al Consejo Nacional de Ciencia y Tecnología (Conacyt) por el apoyo otorgado (228993/211528).

\section{REFERENCIAS}

Aguilar-Rodríguez, S. y J. Barajas-Morales. 2005. Anatomía de la madera de especies arbóreas de un bosque mesófilo de montaña: un enfoque ecológico-evolutivo. Boletín de la Sociedad Botánica de México 77:51-58.

Babos, K. y L.J.C. Cumana. 1992. Xylotomical study of some Venezuelan tree species (Mimosaceae I-IV). Acta Botánica 37(1-4):183-238.

Barajas-Morales, J. y C. León-Gómez. 1989. Anatomía de maderas de México: especies de una selva baja caducifolia. Publicaciones Especiales del Instituto de Biología, UNAM 1:79-80. Baretta-Kuipers, T. 1981. Wood anatomy of Leguminosae: its relevance to taxonomy. In: Polhill R.M. y P.H. Raven, eds. Advances in Legume Systematics. 2:677-705. Royal Botanic Gardens, Kew.

Barneby, R.C. 1991. Sensitivae Censitae. A description of the genus Mimosa L. (Mimosaceae) in the New World. Memoirs of New York the Botanical Garden 65:1-835.

Caccavari, M.A. 1985. Granos de polen de las Leguminosas de la Argentina IV. Género Mimosa. Boletín de la Sociedad Argentina de Botánica 24(1-2):151-167.

Caccavari, M.A. 1986. Estudio de los caracteres del polen de las Mimosa-Lepidotae. Pollen et Spores 28(1):29-42.
Caccavari, M.A. 1987. Estudio de los caracteres del polen de Mimosa-Glanduliferae, VII, Simposio Argentino de paleobotánica y Palinología, Actas:141-144.

Camargo-Ricalde, S.L. 1997. Aspectos de la biología del “tepescohuite", Mimosa tenuiflora (Leguminosae), en México. Tesis de Maestría en Ciencias, Facultad de Ciencias, UNAM, México. 111 p.

Camargo-Ricalde, S.L. 2000. Descripción, distribución, anatomía, composición química y usos de Mimosa tenuiflora (Fabaceae-Mimosoideae) en México. Revista de Biología Tropical 48:939-954.

Camargo-Ricalde, S.L., R. Grether, A. Martínez-Bernal, V. García-García y S. Barrios-del-Rosal. 2001. Especies útiles del género Mimosa (Fabaceae-Mimosoideae) en México. Boletín de la Sociedad Botánica de México $68: 33-44$

Camargo-Ricalde S.L., I. Reyes-Jaramillo y N.M. Montaño. 2010a. Forestry insularity effect of four Mimosa L. species (Leguminosae-Mimosoideae) on soil nutrients of a Mexican semiarid ecosystem. Agroforestry Systems 80:385-397.

Camargo-Ricalde, S.L., N.M. Montaño, I. Reyes-Jaramillo, C. Jiménez-González y S.S. Dhillion. 2010b. Effect of mycorrhizae on seedlings of six endemic Mimosa L. species (Leguminosae-Mimosoideae) from the semi-arid Tehuacán-Cuicatlán Valley, Mexico. Trees-Structure and Function 24:67-78.

Carlquist, S. 1982. Wood anatomy of Onagraceae: further species, root anatomy, significance of vestured pits and allied structures in dicotyledons. Annals of the Missouri Botanical Garden 69:755-769.

Carnieletto, C. y J.N.C. Marchiori. 1993. Anatomia da madeira de Mimosa eriocarpa Benth. Ciência Florestal, Santa Maria 3(1):107-120.

Chattaway, M. 1956. Crystals in wood tissues II. Tropical Woods 104:100-124.

Chehaibar, M.T. 1988. Estudio taxonómico de la serie Xantiae y especies afines del género Mimosa (Leguminosae), Tesis de Maestría en Ciencias, Facultad de Ciencias, UNAM, México. 107 p. 
Chehaibar, M.T. y R. Grether. 1990. Anatomía de la madera de algunas especies del género Mimosa (Leguminosae). Boletín de la Sociedad Botánica de México 50:3-17.

Choat, B., S. Jansen, M.A. Zwieniecki, E. Smets y N.M. Holbrook. 2004. Changes in pit membrane porosity due to deflection and stretching: the role of vestured pits. Journal of Experimental Botany 55:1569-1975.

Cozzo, D. y L.Q. Cristiani. 1950. Los géneros de fanerógamas argentinas con estructura leñosa estratificada. Revista del Museo Argentino de Ciencias Naturales, Bernardino Rivadavia, Ciencias Botánicas 1(8):363-405.

Cozzo, D. 1951. Anatomia del leno secundario de las Leguminosas Mimosoideas y Caesalpinoideas Argentinas, silvestres y cultivadas. Revista del Museo Argentino de Ciencias Naturales, Bernardino Rivadavia, Ciencias Botánicas 2:63-146.

De Oliveira, E., V.B. Rocha, P.A. Santos, L.R.M. Della, A.M.M. Ladeira y C.A. de Cássia Oliveira. 2006. Estrutura antômica da madeira e qualidade do carvÃo de Mimosa tenuiflora (Willd.) Poir. Revista Árvore 30(2):311-318.

Espinoza-de Pernía, N., O.Z. Araque y H.W.J. León. 1998. Cristales de la madera de Schizolobium amazonicum y $S$. parabybum (Caesalpinoideae-Leguminosae). Revista Forestal Venezolana 42(1):9-13.

Evans, J.A., P.E. Gasson y G.P. Lewis. 2006. Wood anatomy of the Mimosoideae (Leguminosae). International Association of Wood Anatomists Journal. Supplement 5. 117 p.

Fabrowski, F.J., G.I.B. Muñiz, M.C.M. Mazza, T. Nakashima, U. Klock, J.C. Possamai, y S. Nisgoski. 2005. Anatomia comparativa da madeira das variedades populares da Bracatinga (Mimosa scabrella Betham). Ciência Florestal 15(1):65-73.

Flores-Cruz, M.F., H.D.S. Lira, A. Martínez-Bernal y M.E. Fraile. 2006. Morfología del polen de Mimosa serie quadrivalves (Leguminosae, Mimosoideae). Acta Botánica Mexicana 77:1-13.

Grether, R. 1978. A general review of the genus Mimosa in México. Bull. Int. Group Study of Mimosoideae 6:45-50. Grether, R., S.L. Camargo-Ricalde y A. Martínez-Bernal. 1996. Especies del género Mimosa (Leguminosae) presentes en México. Boletín de la Sociedad Botánica de México 58:149-152.
Grether, R. 2000. Nomenclatural changes in the genus Mimosa (Fabaceae, Mimosoideae) in Southern Mexico and Central America. Novon 10:29-37.

Heringer, E.P. y J.E. De Paula. 1979. Um novo par vicariante: Mimosa schomburgkii Benth. e Mimosa artemisiana Heringer \& Paula sp. nov. (Floresta Atlántica). In: Anais Congresso Nacional de Botanica do Brasil 30, Campo Grande, Ms. Sociedad Botánica de Brasil, Anais Sociedad Botánica de Brasil: 75-82.

Hintze, J. 2001. Number Cruncher Statistical System (NCSS). IAWA (International Association of Wood Anatomy). 1989. IAWA list of microscopic features for hardwood identification. IAWA Bulletin n.s. 10(3):219-332.

Johansen, D.A. 1940. Plant microtechnique. McGraw-Hill. Nueva York. 523 p.

Kribs, D. 1935. Salient lines of structural specialization in the wood rays of dicotyledons. Botanical Gazzetta 96:547-557.

Kribs, D.E. 1968. Commercial foreign woods on the American marquet. Dover Publications. Inc. New York. 241 p.

Lewis, G.P., B. Schrire y M. Lock, eds. 2005. Legumes of the World. Royal Botanic Gardens, Kew. 592 p.

Maccari, A. y J.N.C. Marchiori. 1994. Estudo anatômico do xilema secundário de Mimosa sparsa Benth. Ciência Florestal 4(1):145-155.

Marchiori, J.N.C. 1982. A estrutura do xilema secudário de Mimosa daleoides Benth. (Leguminosae-Mimosoideae). Ciência e Natura 4:107-113.

Marchiori, J.N.C. 1985. Anatomia da madeira de Mimosa cruenta Benth. (Leguminosae-Mimosoideae). Ciência e Natura 7:73-81.

Marchiori, J.N.C. 1993. Anatomia da madeira e casca do maricá, Mimosa bimucronata (DC.) O. Kuntze. Ciência Florestal 3(1):85-106.

Marchiori, J.N.C. 1996. Anatomia do xilema secundário de Mimosa incana (Spreng.) Benth. Ciência Florestal 6(1):53-63.

Marchiori, J.N.C y B.G. Muñiz. 1997. Estudo anatômico do xilema secundário de Mimosa trachycarpa Benth. Ciência Rural 27(2):223-228.

Metcalfe, C. y I. Chalk. 1979. Anatomy of the dicotyledons. I: Systematic anatomy of the leaf and stem, with a brief history of the subject. Clarendon Press. Oxford. 276 p. 
Moglia, G. y A. Giménez. 1998. Rasgos anatómicos característicos del hidrosistema de las principales especies arbóreas de la región chaqueña argentina. Investigación Agraria 7:53-71

Montaño-Arias, S.A. 2010. Anatomía comparada de la madera de especies arbóreas mexicanas del género Mimosa Sección Batocaulon (Leguminosae). Tesis de Maestría. Universidad Autónoma Metropolitana, Unidad Iztapalapa. México, D.F. 141 p.

Montaño-Arias, S.A., S.L. Camargo-Ricalde y C. De La PazPérez. 2013. Ecoanatomía de los elementos de vaso de la madera de cinco especies del género Mimosa (Leguminosae-Mimosoideae). Botanical Sciences 91(1):1-10.

Moreno-Calles, A. y A. Casas. 2010. Agroforestry systems: restoration of semiarid zones in the Tehuacán Valley, Central Mexico. Ecological Restoration 28:361-368.

Pavón, N.P., J. Ballato-Santos y C. Pérez-Pérez. 2011. Germinación y establecimiento de Mimosa aculeaticarpa var. biuncifera (Fabaceae-Mimosoideae). Revista Mexicana de Biodiversidad 82:653-661.

Record, B.L. y R.W. Hess. 1943. Timbers of the world. Yale University Press. New Haven. EUA. 293-294 p.

Rzedowski, J. 1991. Diversidad y orígenes de la flora fanerogámica de México. Acta Botánica Mexicana 14:3-21.

Simon, M. F., R. Grether, L. P. de Queiroz, T. E. Sarkinen, V. F. Dutra y C. E. Hughes 2011. The evolutionary history of
Mimosa (Leguminosae): towards a phylogeny of the sensitive plants. American Journal of Botany 78:1201-1221.

Sokal, R. y F. Rohlf. 1995. Biometry. Freeman and Company. San Francisco, C.A. 832 p.

Sosa, V. y P. Dávila. 1994. Una evaluación del conocimiento florístico de México. Annals of the Missouri Botanical Garden 81:749-757.

Sousa, S., M., M. Ricker, y H.M. Hernández. 2001. An index for the tree species of the family Leguminosae in Mexico. Harvard Papers in Botany 6(1):339-365.

Sousa, S.M., Ricker, M. y Hernández, H.M. 2003. An index for the tree species of the family Leguminosae in Mexico. Harvard Papers in Botany 7(2):381-398.

Tortorelli, L. 1956. Maderas y bosques argentinos. ACME. Buenos Aires. 910 p.

Zindler-Frank, E. 1987. Calcium oxalate crystals in legumes. In: E. Stirton, ed. Advances in Legume Systematics 3. Royal Botanic Gardens, Kew. p:279-316.

Manuscrito recibido el 2 de julio de 2015 .

Aceptado el 11 de febrero de 2016

Este documento se debe citar como:

Montaño-Arias, S.A., S.L. Camargo-Ricalde y R. Grether. 2016. Anatomía de la madera de tres especies de Mimosa (LeguminosaeMimosoideae) distribuidas en México. Madera y Bosques 22(1):191-202. 